\title{
PERFIL EPIDEMIOLÓGICO DAS PESSOAS ATENDIDAS POR INTOXICAÇÃO EXÓGENA EM UMA UNIDADE DE PRONTO ATENDIMENTO
}

\section{EPIDEMIOLOGICAL PROFILE OF PEOPLE SERVED BY EXOGENOUS POISONING IN AN EMERGENCY CARE UNIT}

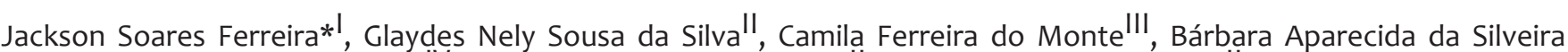 \\ BeserralV , Salmana Rianne Pereira Alves" ", Adelson Francisco Ferreirall
}

\begin{abstract}
Resumo. A intoxicação é a apresentação clínica de efeitos prejudiciais, por interação com substâncias químicas e quando relacionada ao adulto, em sua maioria, a intoxicação exógena está associada às tentativas de suicídio. A pesquisa objetivou investigar o perfil das pessoas atendidas por intoxicação exógena em uma Unidade de Pronto Atendimento. Trata-se de uma pesquisa documental retrospectiva, com abordagem quantitativa, realizada em prontuários de pacientes atendidos de novembro de 2018 a julho de 2019, por intoxicação exógena, em uma Unidade de Pronto Atendimento de João Pessoa, Paraíba, aprovada pelo Comitê de Ética e Pesquisa, sob o CAAE: 20669019.6.0000.5179. Após investigar todos os atendimentos que aconteceram no período e excluídos os prontuários com rasuras e grafias incompreensíveis, a amostra foi composta por 77 prontuários. Como resultado da pesquisa, observou-se $42,8 \%$ com idade entre 19 e 30 anos, sendo 55,8\% dos atendidos do gênero feminino. 57,1\% tem nível médio de escolaridade, $31,2 \%$ eram estudantes e $63,6 \%$ solteiros. Identificou-se $57,1 \%$ de intoxicação por medicações, sendo que $80,5 \%$ não apresentam transtorno mental; $90,9 \%$ dos intoxicados não fazem uso de medicação alopática e $87 \%$ não tem acompanhamento com especialista. Constatou-se que a principal causa da intoxicação exógena se deu através da violência autoprovocada por medicação, mas que nem sempre se tem relação com transtornos mentais. Entretanto, observou-se, através da análise dos resultados obtidos por meio dos prontuários, a prevalência da depressão, com hegemonia de casos em mulheres.
\end{abstract}

PALAVRAS-CHAVE: Envenenamento. Perfil Epidemiológico. Substância Nociva. Suicídio. Socorro de Urgência.

\begin{abstract}
Intoxication is the clinical presentation of harmful effects, due to interaction with chemical substances and when related to adults, in most cases, exogenous intoxication is associated with suicide attempts. The research aimed to investigate the profile of people assisted by exogenous intoxication in an Emergency Care Unit. This is a retrospective documentary research, with a quantitative approach, performed on medical records of patients assisted from November 2018 to July 2019 for exogenous intoxication in an Emergency Care Unit in João Pessoa, Paraíba, approved by the Research Ethics Committee under protocol number CAAE: 20669019.0000.5179. After investigating all the consultations that occurred in the previously mentioned period and excluding the medical records with incomprehensible erasures and handwritings, the sample consisted of 77 medical records. As a result of the research, $42.8 \%$ were aged between 19 and 30 years old, $55.8 \%$ were female, $57.1 \%$ had graduated high school, $31.2 \%$ were students, and $63,6 \%$ were single. $57.1 \%$ corresponded to intoxication by medication, and $80.5 \%$ did not report any mental disorder; $90.9 \%$ did not make use of allopathic medication and $87 \%$ were not under treatment with a specialist. It was found that the main cause of exogenous intoxication was self-harm caused by medication, which is not always related to mental disorders, however, through the analysis of the results obtained from medical records, the prevalence of depression was observed, with a female hegemony of cases.
\end{abstract}

KEYWORDS: Poisoning. Epidemiological Profile. Harmful Substance. Suicide. Emergency Relief.

Enfermeiro. Faculdade de Enfermagem Nova Esperança - FACENE. Departamento de Enfermagem. CEP: 58067-695, João Pessoa, Paraíba, Brasil.

II Enfermeiro(a). Mestre em Saúde da Família. Docente do Curso de Enfermagem da Faculdade de Enfermagem Nova Esperança. Departamento de Enfermagem. CEP: $58067-695$, João Pessoa, Paraíba, Brasil. ORCID ID: 0000-0002-6564-657X; 0000-0002-4472-2289; 0000-0002-9336-7922.

III Graduanda. Curso de Enfermagem da Faculdade de Enfermagem Nova Esperança. Departamento de Enfermagem. CEP: 58067-695, João Pessoa, Paraíba, Brasil. ORCID ID: 0000-0002-5658-3458

IV Enfermeira. Especialista em Qualidade e Segurança no Cuidado ao Paciente. Faculdade de Enfermagem Nova Esperança. Departamento de Enfermagem. CEP: 58067-695, João Pessoa, Paraíba, Brasil. ORCID ID: $0000-0003-4082-2132$ 


\section{INTRODUÇÃO}

A intoxicação é a manifestação clínica de efeitos prejudiciais ao organismo, consequentes de interações com substâncias químicas, representando de $5 \%$ a $10 \%$ dos atendimentos no setor da emergência. Além disso, o envenenamento é uma das principais causas que resultam na morte acidental de crianças, porém quando se refere ao adulto é mais frequente observar tentativas de suicídio. ${ }^{1}$

No Brasil, a intoxicação exógena também é uma das formas utilizadas para tentativas e suicídio. As substâncias que têm relação com $70 \%$ dos casos, são medicamentos e pesticidas, ${ }^{2}$ prevalecendo, também, o uso de produtos de limpeza como opção de substância utilizada. A intoxicação exógena quando comparada a outros métodos no quesito de tentativas exitosas, a exemplo do enforcamento, obtém menos incidência, pois possui altos índices de sobrevivência. 3

Observa-se assim, a preferência e até mesmo um padrão de uso de substâncias de fácil acesso. Entretanto, o coeficiente de mortalidade por suicídio no Brasil é de 5,3 mortes por 100 mil habitantes. 4

Existe um amplo arsenal de fatores influenciadores de comportamentos suicidas,

\section{MATERIAL E MÉTODOS}

Trata-se de uma pesquisa documental retrospectiva com abordagem quantitativa realizada em Unidade de Pronto Atendimento (UPA), em João Pessoa, no estado da Paraíba. A escolha ocorreu por ser um serviço específico para atender as demandas de urgências, inclusive de pessoas vítimas de intoxicação exógena. entre eles os mais apontados com frequência são, depressão, alcoolismo, perdas recentes, conflitos familiares, sentimento de desamparo e transtornos mentais. Porém, destaca-se que pacientes psiquiátricos apresentam maior probabilidade para cometer suicídio, quando comparado a população geral, sendo assim considerado predisponente de manifestar comportamentos de autodestruição. 2,5

A maioria dos estudos realizados são feitos com base em suicídios que obtiveram êxito, no entanto, é sabido que a intoxicação exógena é um dos meios com menos eficácia quando comparada a outros métodos. 3 Nesse sentido, a pesquisa objetivou investigar o perfil das pessoas atendidas por intoxicação exógena em uma Unidade de Pronto Atendimento, auxiliando na agregação de informações sobre a temática em diversas áreas para que profissionais que tenham acesso a esse perfil atuem de forma humanizada, tendo em vista que quando se define um perfil, se torna mais fácil elaborar estratégias de controle. Desta forma, a presente pesquisa obtém como questão norteadora: Qual o perfil das pessoas atendidas na Unidade de Pronto Atendimento por intoxicação exógena?

A seleção da amostra foi feita a partir da investigação dos prontuários de pacientes atendidos na UPA, no período de novembro de 2018 a julho de 2019, que totalizou 72.272 atendimentos, sendo composta por todos os prontuários, cujo motivo de atendimento foi a intoxicação exógena, com um total de 77 prontuários. Foram excluídos os prontuários 
com rasuras e grafias incompreensíveis.

$O$ instrumento utilizado para a coleta de dados foi um roteiro com questões norteadoras, elaborado com base no modelo dos prontuários de atendimento do serviço onde a pesquisa foi realizada, tendo como variáveis: idade, gênero, escolaridade, profissão, estado civil, substâncias químicas utilizadas pelos pacientes, presença de transtorno mental, tipo de transtorno, uso de remédio alopático-qual?-eacompanhamento com especialista - qual? -

A coleta de dados foi efetivada no mês de agosto de 2019, em horários previamente agendados com o responsável pelo setor onde ficam arquivados os prontuários, após a autorização da Secretaria Municipal de Saúde de João Pessoa, instituição responsável pela unidade de saúde e aprovação do Comitê de Ética em Pesquisa (CEP) da Faculdade de Enfermagem Nova Esperança, sob o número de CAAE: 20669019.6.0000.5179.

\section{RESULTADOS E DISCUSSÃO}

No período de novembro de 2018 a julho de 2019 foram notificadas na UPA 77 vítimas de intoxicação exógena, com caracterização social conforme apresentado na Tabela 1. No tocante a faixa etária, as pessoas que têm entre 19 a 30 anos representam a maior porcentagem de casos, sendo $42,8 \%$ dos registrados por intoxicação exógena. No que se refere ao gênero, o feminino se sobressaiu com $55,8 \%$ dos casos.

TABELA 1: Caracterização social dos pacientes atendidos por intoxicação exógena na UPA em João Pessoa, PB, Brasil, entre novembro de 2018 a julho de 2019

\begin{tabular}{cccc}
\hline Variáveis & & N & $\%$ \\
\hline \multirow{4}{*}{ Idade } & 2 a 10 anos & 3 & 3,9 \\
& 11 a 18 anos & 23 & 29,9 \\
& 19 a 30 anos & 33 & 42,8 \\
& 31 a 45 anos & 14 & 18,2 \\
& 46 a 60 anos & 4 & 5,2 \\
\hline \multirow{2}{*}{ Gênero } & Masculino & 34 & 44,2 \\
& Feminino & 43 & 55,8 \\
\hline \multirow{4}{*}{ Escolaridade } & Ensino Fundamental & 9 & 11,7 \\
& Ensino Médio & 44 & 57,1 \\
& Ensino Superior & 8 & 10,4 \\
& Não Informado & 16 & 20,8 \\
\hline \hline \multirow{4}{*}{ Profissão } & Estudante & 24 & 31,2 \\
& Autônomo & 6 & 7,8 \\
& Assalariado & 23 & 29,9 \\
& Desempregado & 4 & 5,2 \\
& Não Informado & 20 & 25,9 \\
\hline \hline \multirow{2}{*}{ Estado Civil } & Solteiro & 49 & 63,6 \\
& Casado & 14 & 18,2 \\
& Não Informado & 14 & 18,2 \\
\hline \hline Total & & 77 & 100 \\
\hline \hline
\end{tabular}

No cenário mundial, a tentativa de suicídio no ano de 2016 foi a segunda maior causa de óbitos entre adultos e jovens com idades entre 15 e 29 anos. $^{6}$ Fato este que corrobora com os dados apresentados anteriormente pelo presente estudo. Nesse contexto, destaca-se na literatura que mulheres realizam mais tentativas de 
suicídio e que os fatores que as deixam mais vulneráveis são violência doméstica, abuso sexual, aspectos culturais, entre outros. 4 Esta disposição feminina pode também ser constatada no presente estudo.

No que concerne ainda a caracterização social, pessoas com ensino médio se sobressaíram entre as atendidas por intoxicação exógena (57,1\%). Quanto a profissão das vítimas de intoxicação exógena, foi observada pouca diferença entre as ocupações, sendo mais presentes em estudantes (31,2\%). No que diz respeito a prevalência de vítimas com estado civil solteiro (63,6\%), podemos pensar na coerência da teoria das necessidades humanas básicas, que é composta por necessidades fisiológicas, de segurança, de amor, de estima e de autorrealização.7

Estas necessidades são relacionadas entre si, fazendo parte do ser humano de forma holística. Quando ocorre desequilíbrio ou insatisfação em uma dessas necessidades prevalecem sinais e sintomas na saúde do indivíduo.7 Dessa forma, observou-se nos resultados da presente pesquisa que os mais altos índices de intoxicação foram identificados em pessoas que possuíam alguma insatisfação nestas necessidades, seja de forma social no caso do estado civil, ou de estima e autorrealização no quesito profissional e de escolaridade.

Correlacionando esses resultados a teoria da adaptação, o comportamento humano é relacionado a modos. O modo de autoconceito tem ligação com a integralidade psíquica e a necessidade básica em que o ser procura equilíbrio mental de acordo com o conceito formado sobre si mesmo. Já modo de interdependência mostra a necessidade das relações humanas, ou seja, interações sociais. Em suma, aspectos que possam mudar o autoconceito do ser (estado civil, escolaridade, ocupação) têm influência sobre seu equilíbrio mental e susceptivelmente ao seu comportamento. ${ }^{8}$

Observa-se na Tabela 2 que 44 $(57,1 \%)$ dos casos de intoxicações exógenas são causados por ingesta de medicações e, em sua maioria, são usados ansiolíticos, antidepressivos e psicotrópicos. Tal prática pode estar relacionada a dependência de drogas, colocando o usuário em posição de vulnerabilidade, tendo em vista consequências como falta de emprego, abandono da escola, o suicídio propriamente dito e demais fatores relacionados as necessidades humanas básicas. 9

No Brasil, prevalece como primeira escolha e obtém relação em $70 \%$ dos casos de intoxicação exógena, o uso de medicamentos e pesticidas, seguidos por produtos de limpeza. 2,10 Em outras pesquisas, também, foi possível observar os medicamentos como principal causa de intoxicações exógenas, seguidos de agrotóxicos e/ou drogas de abuso. ${ }^{11,12}$

TABELA 2: Substâncias químicas utilizadas pelas pessoas atendidas por intoxicação exógena na UPA em João Pessoa, PB, Brasil, entre novembro de 2018 a julho de 2019

\begin{tabular}{ccc}
\hline Variáveis & N & $\%$ \\
\hline \hline Medicação & 44 & $\mathbf{5 7 , 1}$ \\
Entorpecente ilícito & 18 & 23,4 \\
Pesticida & 10 & 13 \\
Produto de limpeza & 2 & 2,6 \\
Não identificado & 3 & 3,9 \\
\hline \hline Total & $\mathbf{7 7}$ & $\mathbf{1 0 0}$ \\
\hline & \multicolumn{2}{c}{ Fonte: Pesquisa direta. }
\end{tabular}


Com base nos resultados obtidos, $80,5 \%$ dos casos não apresentam transtorno mental. Dos que possuem algum transtorno mental, 66,6\% são representados pela depressão como pode ser visto na Tabela 3.
No que concerne ao uso de remédios alopáticos, 90,9\% das vítimas não utilizavam. No entanto, dos que fazem uso, o Rivotril (57,1\%) é a medicação com maior frequência de uso.

TABELA 3: Dados referentes a transtornos mentais e tratamento entre as pessoas atendidas por intoxicação exógena na UPA em João Pessoa, PB, Brasil, entre novembro de 2018 a julho de 2019

\begin{tabular}{|c|c|c|c|}
\hline Variáveis & & $\mathbf{N}$ & $\%$ \\
\hline \multirow{2}{*}{ Transtorno Mental } & não & 62 & 80,5 \\
\hline & $\operatorname{sim}$ & 15 & 19,5 \\
\hline \multirow{4}{*}{ Tipo de transtorno } & Depressão & 10 & 66,6 \\
\hline & Bipolaridade & 1 & 6,7 \\
\hline & Ansiedade & 3 & 20,0 \\
\hline & Alucinação & 1 & 6,7 \\
\hline \multirow{2}{*}{ Uso de remédio alopático } & não & 70 & 90,9 \\
\hline & $\operatorname{sim}$ & 7 & 9,1 \\
\hline \multirow{3}{*}{ Remédio } & Rivotril & 4 & 57,1 \\
\hline & Carbamazepina & 2 & 28,6 \\
\hline & Não Informado & 1 & 14,3 \\
\hline \multirow{2}{*}{ Acompanhamento com especialista } & não & 67 & 87,0 \\
\hline & $\operatorname{sim}$ & 10 & 13,0 \\
\hline \multirow{4}{*}{ Especialista } & Psiquiatra & 5 & 50,0 \\
\hline & Psicólogo & 1 & 10,0 \\
\hline & Neurologista & 1 & 10,0 \\
\hline & Não Informado & 3 & 30,0 \\
\hline
\end{tabular}

Tais resultados salientam a desconstrução do estigma de relacionar a tentativa de suicídio apenas com transtornos mentais. ${ }^{6}$ Contudo, estudos apontam que os transtornos mentais deixam os indivíduos mais susceptíveis e estão presentes na maioria dos casos de suicídio, sendo a depressão um dos principais desencadeantes para comportamentos suicidas. ${ }^{2,}$, 13

Os resultados de um estudo realizado por Moreira, ${ }^{14}$ que objetivou analisar as evidências científicas acerca da presença de transtornos mentais e o risco de suicídio em usuários de substâncias psicoativas, mostram que o principal transtorno mental associado às tentativas de suicídio, são os classificados como CID 10 - transtornos de humor, com ênfase na depressão, se apresentando com maior frequência em mulheres. ${ }^{15}$

As implicações individuais e coletivas do suicídio são analisadas a partir de casos registrados como auto violência. Devem ser recordados fatores que influenciam na negação de registros, tais como, estigma social e familiar, aspectos religiosos, entre outros. ${ }^{16}$

O processo de retirada da própria vida deve ser compreendido como um momento de aflição, quase sempre associado a dificuldade de administrar a própria vida. Entretanto, é possível pensar em fatores associados, como tentativas anteriores, isolamento social, histórico familiar, tanto de doenças mentais como de abuso, dentre outros elementos biológicos e socioeconômicos. ${ }^{17}$

Destarte, sabe-se que quando a depressão é diagnosticada, busca-se o tratamento de imediato, seja através de psicoterapia e/ou farmacoterapia.

Nesse contexto, a pesquisa mostrou que prevalece aqueles que não fazem nenhum acompanhamento com especialista, sendo psiquiatria (50\%) a especialidade mais procurada dos que buscam ajuda profissional. Esse pequeno percentual apresentado 
pode estar associado ao preconceito social referente a tudo aquilo que esteja associado a transtornos mentais, implicando, assim, na

\section{CONCLUSÃO}

Nos últimos anos, o número de atendimentos por intoxicação exógena cresce cada vez mais e, em sua maioria, esses casos têm como principal causa a tentativa de suicídio, que não necessariamente se apresenta associada a um transtorno mental. Destaca-se que as mulheres se apresentam mais vulneráveis com idade entre 19 a 30 anos.

Embora haja limitações no estudo como, por exemplo, a escassez de estudos científicos que abrangem a temática, a presente pesquisa se mostra importante

\section{REFERÊNCIAS BIBLIOGRÁFICAS}

1. Volpato $A C B$, Silva ES. Primeiros Socorros. $1^{\mathrm{a}}$ ed. São Paulo: Martinari; 2017.

2. Santos SA, Legay LF, Aguiar FP, Lovisi GM, Abelha L, Oliveira SP. Tentativas e suicídios por intoxicação exógena no Rio de Janeiro, Brasil: análise das informações através do linkage probabilístico. Cad. Saúde Pub. 2014; 30(5): 105766.

3. Santos MSP, Silva TPS, Pires CMC, Ramos PGX, Sougey EB. Identificação de aspectos associados à tentativa de suicídio por envenenamento. J. bras. psiquiatr. 2017; 66(4): 197-202.

4. Veloso C, Monteiro CFS, Veloso LUP, Figueiredo MLF, Fonseca RSB, Araújo TME, et al. Violência autoinfligida por intoxicação exógena em um serviço de urgência e emergência. Rev. Gaúcha Enferm. 2017; 38(2): 1-8. melhoria da qualidade de vida das pessoas que superam um quadro de intoxicação exógena.

para a comunidade acadêmica, em geral, pois fornece dados que contribuem para o conhecimento social e de órgãos responsáveis para ações que forneçam a redução de casos tratados nesta pesquisa, associando as demais pesquisas já realizadas que contribuíram para a construção desta. Por isso, faz-se necessária a continuidade de pesquisas relacionadas ao tema para uma maior rede de informações sobre intoxicações exógenas, seja ela acidental ou autoprovocada.

5. Pires MCC, Raposo MCF, Sougey EB, Bastos Filho OC, Silva TS. Indicadores de risco para tentativa de suicídio por envenenamento: um estudo caso-controle. J. bras psiquiatr. 2015; 64(3): 193-99.

6. Organização Mundial de Saúde. 2018 Perto de 800 mil pessoas cometem suicídio todos os anos. ONU NEWS. [Publicação na web]. 2018 [acesso em 21 mar 2019]; Disponível em: https:// news.un.org/pt/story/2018/09/1637061.

7. Marçal JA. Nursing Process. Rev. enferm. UFPE online. 2017; 11(11): 4533-34.

8. George JB. Teorias de Enfermagem: Os Fundamentos à Prática Profissional. 4 ed. Porto Alegre: Artmed; 2000.

9. Moreira DFN, Santos IL, Azevedo BMEP, Araújo DD, Gusmão ROM. Diagnósticos de 
Enfermagem Identificados em usuários de álcool e outras drogas. Enfermagem em Foco. 2018; 10(5): 103-08.

10. Oliveira EM, Félix TA, Mendonça CBL, Souza $D R$, Ferreira GB, Freire MA, et al. Tentativa de suicídio por intoxicação exógena: contexto de notificações compulsórias. Rev Eletrônica Gestão \& Saúde. 2015; 6(3): 2497-11.

11. Takemoto AY, Bussolotto F. Prevalência de intoxicação exógena em um município do centrooeste do Paraná. Faculdade Guairacá. Trabalho de Conclusão de Curso de Enfermagem. 2019.

12. Carvalho FSA, Mororó WMD, Alencar YCA, Sette RBT, Sousa MNA. Intoxicação exógena no estado de Minas Gerais, Brasil. Ciência \& Desenvolvimento-Revista Eletrônica da FAINOR. 2017; 10(1): 172-84.

13. Ferreira ML, Vargas MAOV, Rodrigues
J, Trenton D, Brehmer LCF, Lino MM. Comportamento suicida e atenção primária a saúde. Enfermagem em Foco. 2018; 9(4): 50-54.

14. Moreira RMM, Oliveira EM, Lopes RE, Lopes MVO, Félix TA, Oliveira LS. Mental disorder and suicide risk in psychoactive substance users: na integrative review. SMAD. Rev. Eletrônica Saúde Mental Álcool Drog. 2020; 16(1): 1-10.

15. Paes MR, Maftum MA, Felix JVC, Mantovani MF, Mathias TAF. Caracterização de pacientes com transtornos mentais de um hospital geral e de ensino. Cogitare Enferm. 2018; 23(2): 1-10. 16. Ribeiro JM, Moreira MR. Uma abordagem sobre o suicídio de adolescentes e jovens no Brasil. Ciênc. Saúde Coletiva. 2018; 23(9):2821-34.

17. Pereira AS, Wilhelm AR, Koller SH, Almeida RMM. Fatores de risco e proteção para tentativa de suicídio na adultez emergente. Ciênc. Saúde coletiva. 2018; 23(11): 3767-77. 